\title{
Effect of gamma radiation on reduction of mycotoxins in black pepper.
}

\begin{abstract}
Gamma ray was applied to reduce mycotoxins, i.e. ochratoxin A (OTA) and aflatoxins B1, B2, G1 and G2 (AFB1, AFB2, AFG1 and AFG2) in black pepper. Response surface methodology (RSM) was applied to evaluate the effect of dose of gamma ray ranging from 0 to $60 \mathrm{kGy}$ and mycotoxin concentration ranging from 10 to $100 \mathrm{ng} \mathrm{g}-1$ on the mycotoxin reduction. The maximum reduction was found at $60 \mathrm{kGy}$ which was $52 \%, 43 \%, 24 \%, 40 \%$ and $36 \%$ for OTA, AFB1, AFB2, AFG1 and AFG2, respectively. Results showed the gamma rays even at $60 \mathrm{kGy}$ were not effective in completely destroying of ochratoxin and aflatoxins.
\end{abstract}

Keyword: Black pepper; Mycotoxin; Gamma ray. 